\title{
Centros que crecen con la comunidad: descubriendo vías para el cambio
}

\author{
Carmen Álvarez Álvarez, Universidad de Cantabria, Santander, España \\ María Verdeja Muñiz, Universidad de Oviedo, Oviedo, España
}

Resumen: En el presente artículo revisamos algunas aproximaciones teóricas y reflexionamos acerca de cómo pueden aprender las escuelas poniendo el acento en la participación de la comunidad y de la propia organización escolar en los procesos de innovación y cambio educativo. Igualmente aportamos algunas experiencias de profesorado que reflexiona sobre su práctica docente y apuesta por una relación dialógica con el alumnado, profesorado y la participación de las familias, como principal herramienta para favorecer el cambio y la mejora de los procesos educativos. Finalmente, desde una mirada crítica y reflexiva apostamos por una escuela democrática y participativa, que contempla la apertura de centros a la comunidad educativa, como una forma de innovación y cambio en las organizaciones educativas para dar respuestas a los cambios sociales, politicos y culturales de nuestras sociedades, siendo clave la participación de todos los miembros en la comunidad educativa

Palabras Clave: Innovación Educativa, Participación, Comunidad Educativa, Organización Escolar, Organizaciones que Aprenden

Abstract: In this article we review some theoretical approaches and reflect on how you can learn schools emphasizing the participation of the community and the school organization in the processes of innovation and educational change. We also provide some experiences of teachers who reflect on his teaching and practice bets for a Dialogic relationship with the students, faculty and the participation of the families, as the main tool to promote change and improvement of the educational process. Finally, a critical and reflexive perspective we are committed to a democratic and participatory school that it contemplates the opening of centers to the educational community, as a form of innovation and change in educational organizations to respond to social, political and cultural changes in our societies, being key to the participation of all members in the educational community.

Keywords: Educational Innovation, Participation, Educational Community, School Oganization, Learning Organizations

\section{Organizaciones que crecen con la comunidad}

$\mathrm{N}$

OS ENCONTRAMOS EN un momento en el que se están produciendo cambios de diferente índole en nuestras sociedades (políticos, económicos, sociales, tecnológicos, cultuales, demográfico, etc.). Esto, ni es nada nuevo, ni nos tendría que sorprender, puesto que la sociedad está en continuo cambio; además consideramos que el hecho de que se produzcan cambios en las sociedades es un ciclo natural de la vida, y puede ser muy positivo. Lo interesante, y la idea que iniciamos este artículo, es la de plantearnos y analizar cómo está respondiendo la escuela ante estos cambios, y lo más

Revista Internacional de Educación y Aprendizaje

Volumen 1, 2013, http://sobrelaeducacion.com/revistas/coleccion/, ISSN 2255-453X

(C) Global Knowledge Academics. Carmen Álvarez Álvarez, María Verdeja Muñiz

Todos los Derechos Reservados. Permisos: soporte@gkacademics.com

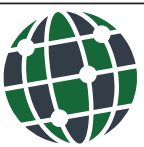


importante aún: ¿cómo está cambiando la escuela para hacer frente a los mismos?, ¿cómo preparar a los futuros ciudadanos/as para convivir en las sociedades de hoy y del mañana?

Santos Guerra (2005) menciona que cada ciudadano tiene que plantearse esta cuestión y, más intensamente, cada profesional que trabaja en una institución educativa. ¿Qué papel desempeña la escuela en la formación de los individuos y en la mejora de la sociedad? ¿A quién beneficia la escuela? ¿Cómo aprende para transformarse en una escuela mejor? Desde el punto de vista de este autor, se percibe la idea de que tanto los profesionales que trabajan en las organizaciones educativas como el resto de los ciudadanos han de estar implicados en la tarea de la formación de los futuros ciudadanos y a todos corresponde preguntarse ¿qué podemos hacer, tanto profesorado como el resto de la comunidad educativa, por mejorar la educación de los futuros ciudadanos?

Para Fernández Enguita (2006), los cambios económicos, sociales y culturales que vivimos requieren pensar y entender la escuela de un modo muy abierto a la comunidad; ya que es un primer punto de encuentro de familias y lugar donde conviven diferentes culturas, convirtiéndose así en uno de los primeros espacios de ciudadanía y por tanto puerta de entrada a la sociedad de acogida. Esto supone un cambio con lo que tradicionalmente se estaba haciendo:

El tránsito a la sociedad de la información, o del conocimiento, implica, por otra parte, que una y otro ya no están confinados a los canales tradicionales: unos pocos que los crean, unos cuantos que los transmiten y el resto que los reciben. Información y conocimiento se crean, acumulan y transmiten hoy en y a través de múltiples organizaciones, grupos y redes entre los cuales la escuela no es sino uno más, aun cuando ocupe un lugar destacado (Fernández Enguita, p. 22).

Es importante distinguir la diferencia entre aprendizaje organizativo, (organizational learning), como apunta Tsang (1997), referido al estudio de los procesos de aprendizaje de (y dentro de) las organizaciones, y el término "organización que aprende" (learning organization), vista como una entidad o tipo ideal de organización, que tiene la capacidad de aprender con eficacia y, por lo tanto, desarrollarse. Nosotros, en el presente artículo hacemos un enfoque de las organizaciones que aprenden desde el "aprendizaje organizacional".

Pero también es importante preguntarse cómo aprenden las escuelas: “Al ser las escuelas instituciones de enseñanza, no habría de parecer descabellado preguntarse cómo éstas aprenden. Sin embargo esta pregunta tan lógica es poco habitual”. (San Fabián, 1996, p. 41).

Diferentes autores ya se han preocupado por estos temas relacionados con los procesos de innovación y cambio en los centros escolares; San Fabián (1996); Martín Bris (2000), Bolívar (2000, 2001), López Yáñez, (2003), Santos Guerra (2005), haciendo popular el concepto de "organizaciones que aprenden", para explicar el carácter dinámico de las organizaciones educativas, analizando al mismo tiempo cómo pueden desarrollarse las escuelas e identificando algunos factores determinantes que influyen en la propia cultura de la organización.

Para Bolívar (2000), una organización aprende cuando, por haber optimizado el potencial formativo de los procesos que tienen lugar en su seno, adquiere una función cualificadora para los que trabajan en ella, al tiempo que está atenta para responder a las demandas y cambios externos. 




Figura 1: El centro educativo como organización que aprende (Bolívar, p.193)

Hay autores como Martín Bris (2000), que centran su atención en el clima o ambiente de trabajo en las organizaciones, siendo uno de los factores determinantes y facilitadores de los procesos organizativos y de gestión las relaciones entre los miembros de la organización, tanto los profesionales, como la comunidad educativa en general. También son fundamentales los procesos de innovación que se generan en las escuelas porque éstos promueven cambios culturales profundos. Compartimos la idea de este autor en cuanto que hace alusión al carácter dinámico de la organización escolar y explica cómo aspectos como la comunicación, la motivación, la confianza, la participación, la planificación, el liderazgo y la creatividad, contribuyen a mejorar el clima de trabajo o el ambiente de la organización y se producen cambios en la propia organización, por lo que está haciendo referencia al carácter dinámico de la misma.

San Fabián (2011) apuesta por la investigación en el campo de la organización escolar, poniendo de manifiesto (y cuestionando) la escasa tradición investigadora en el campo de la organización escolar, señalando entre otros algunos condicionantes tales como: la dependencia de las organizaciones no escolares, la excesiva atención a los enfoques formales, los enfoques positivista, el extrañamiento docente respecto a lo organizativo.

La investigación educativa ha marginado el contexto organizacional de la enseñanza y ha reforzado las perspectivas funcionalistas: una ingenua -funcionalismo pedagógico-, que piensa que las instituciones educativas están organizadas de la mejor manera posible para cumplir sus objetivos, y otra determinista -funcionalismo sociológico-, según la 
cual los esfuerzos en el cambio organizativo se muestran de manera poco relevante en los resultados (San Fabián, pp. 56-57).

Hay otros autores, como López Yáñez (2003), que también acusan el déficit teórico en los estudios sobre las organizaciones sociales en general, y las educativas en particular, y apuntan que la Organización Escolar como disciplina sigue estando dominada por los enfoques prescriptivos y normativos sobre los descriptivos e interpretativos, dificultando el crecimiento del campo y su conocimiento en profundidad. López Yáñez apuesta por una teoría del aprendizaje organizativo que permita tener un mejor conocimiento y comprender cómo se producen los procesos de innovación y cambio en la propia institución. Para este autor, la innovación es un proceso de aprendizaje institucional:

La innovación educativa aparecería básicamente como un proceso de aprendizaje institucional, en donde los aspectos estratégicos tendrían tanta importancia como los simbólicos, y en donde el éxito del proceso dependería de la habilidad de los agentes de cambio para construir una espiral creativa que sintetice la lectura externa del cambio y la lectura interna (López Yáñez, p.148).

San Fabián (2011) apunta, en la línea de Stenhouse, al "profesor investigador" cuando hace alusión a que la investigación mejoraría las organizaciones educativas, cuando las organizaciones asuman la investigación como función propia. Compartimos la idea del profesor como investigador, que reflexiona sobre su propia práctica, reformulando planteamientos teóricos, llevándolos a la práctica docente y mejorándola. Para ello una herramienta ideal es el diálogo con el alumnado, las familias y el trabajo colaborativo con el profesorado y con la sociedad en general. Como apuntan Imbernón y Bonafé (2008), "cambiar la práctica educativa significa cambiarse a sí mismo, como profesional, cambiar el contexto educativo, el lugar de desempeño, y hacerlo conjuntamente con los demás mediante el diálogo, la negociación y la colaboración".

Para esto, desde nuestro punto de vista, son fundamentales dos cuestiones: 1) Implicar a todos los/as ciudadanos/as y a la comunidad educativa en general en la tarea de educar, pudiendo esta implicación hacerse en diferentes momentos y formas de participación y 2) Abrir los centros a la comunidad educativa para favorecer los procesos de participación, cambio e innovación.

Martínez González y San Fabián (2002) también hacen referencia a la importancia que tiene que la escuela facilite la participación de la comunidad educativa y apostando por compartir los recursos que nos ofrece el entorno y trabajar de una forma coordinada, construyendo así redes educativas.

Los centros deben promover un proyecto cultural comunitario, que recoja propuestas de coordinación de los servicios educativos, sociales y de salud de una comunidad, así como acciones conjuntas con recursos educativos del entorno. Ello implica abrir la escuela al entorno y permitir el uso de las instalaciones por parte de grupos y asociaciones del barrio, implicar a estos grupos en la realización de propuestas educativas dentro y fuera del centro en colaboración con el profesorado y los padres. No se trata tanto de ampliar la red escolar como de construir redes educativas (Martínez González y San Fabián, p. 10). 
En la misma dirección apunta Bolívar Ruano (2012), cuando hace hincapié en que se debe trabajar en la construcción de una comunidad profesional que se caracteriza, entre otros, por un propósito común, un trabajo en equipo y de colaboración y la responsabilidad colectiva entre el personal por el aprendizaje. Al mismo tiempo González González (2011), pone el acento en el centro escolar, como contexto de relaciones para el aprendizaje de la convivencia y para la socialización de los estudiantes:

Los centros escolares en cuanto organizaciones, constituyen un contexto clave para el desarrollo de procesos curriculares y de enseñanza-aprendizaje, y para la socialización de los estudiantes. Los valores, los principios y propósitos sobre los que el centro asienta su actuación y funcionamiento, las estructuras organizativas con las que cuenta, las relaciones entre sus miembros y con la comunidad escolar y los procesos organizativos que se llevan a cabo en él conforman las condiciones organizativas en las que se llevará a cabo la actividad docente de los profesores y el aprendizaje de los alumnos. (González González, pp. 41-42).

En España, contamos con la iniciativa de las Comunidades de Aprendizaje que han contribuido a transformar la dinámica de sus escuelas para convertirlos en espacios más participativos, abiertos y plurales, soñando toda la comunidad el centro deseado y poniéndose manos a la obra en su construcción solidaria (Elboj, Puigdellívol, Soler y Valls, 2006), diluyéndose cada vez más la tendencia a separar lo que es centro y lo que es comunidad, ofreciendo un modelo teórico-práctico deseable para todas las escuelas. Una de las características principales de las comunidades de aprendizaje es su apuesta por el aprendizaje dialógico mediante la implementación de actuaciones educativas "de éxito", como los grupos interactivos, la formación de familias, las bibliotecas tutorizadas, las tertulias literarias, etc. y el diálogo igualitario como principio, que permite desarrollar un esfuerzo solidario entre los miembros de la comunidad encaminado a lograr la igualdad educativa de todas las alumnas y alumnos. Los retos que se plantea el proyecto de comunidades de aprendizaje exigen la participación de la comunidad en todos los espacios, incluida el aula. El diálogo en la escuela tiene que abarcar al conjunto de la comunidad de aprendizaje, incluyendo familiares, voluntariado, profesionales, alumnado y profesorado. Todas las personas influyen en el aprendizaje y todos y todas deben planificarlo conjuntamente. El centro educativo se transforma en un espacio en el que las familias comparten sus preocupaciones, resuelven sus dudas, encuentran soluciones conjuntas a los problemas de su vida diaria y, sobre todo, se forman. (Flecha, Padrós y Puigdellívol (2003, p.5).

\section{Participación en las organizaciones educativas: una forma de innovación}

En términos estrictos del discurso no es fácil decir nada nuevo sobre las relaciones entre educación y participación. Sin embargo pensamos que en ocasiones, las ganas de innovación y cambio en las organizaciones educativas han dejado al margen otros ámbitos, como es la participación, pasando así a estar en un segundo plano, sin darnos cuenta de la importancia y sentido de la participación, para que esas innovaciones y cambios sean posibles. 
Por un lado, vivimos una época donde la participación no parece formar parte de las preocupaciones educativas actuales, definidas más bien como problemas técnicos en términos de calidad, eficiencia o implantación de nuevas tecnologías, y que se resuelven en el ámbito de las opciones individuales. Por otro lado, la participación forma parte del discurso oficial como algo asumido, que se da por supuesto y, por tanto, que no necesita revisarse, lo que impide que se discuta sobre ella (San Fabián, 2005, p. 179).

Compartimos esta idea, y pensamos que tal sea porque se ha restado importancia a la participación de las familias y actualmente está en un segundo plano, cuando desde nuestro punto de vista es algo fundamental que puede contribuir a los procesos de innovación y cambio dentro de la organización. Cuando hablamos de innovación y cambio en las organizaciones, estamos hablando de algo mucho más complejo y profundo que trasciende la puesta en marcha de proyectos, programas educativos u otro tipo de propuestas. La participación de las familias en los centros educativos, es en sí, una innovación educativa que pude mejorar y cambiar las organizaciones educativas.

En ocasiones, se ha malentendido el término de innovación, y se ha pasado a pensar que innovar es hacer proyectos, reformas, desarrollar programas educativos, etc., cuando innovar en realidad es algo más profundo y duradero en el tiempo. Malinterpretar el concepto de innovación ha producido un efecto contrario, ocasionando al mismo tiempo más desconcierto. En ese sentido hay autores como Fullan (2002), que considera que una de las principales dificultades para la mejora de las escuelas de los países ricos occidentales no es la ausencia de innovación, sino más bien la presencia de demasiados proyectos novedosos inconexos, episódicos, fragmentados y adornados de forma superflua.

En la actualidad la escuela no es una organización que aprenda. Olas irregulares de cambio, proyectos ocasionales, la fragmentación del esfuerzo y una sobrecarga agotadora es lo que componen la mayoría de las escuelas. La mayor parte de los esfuerzos de cambio son erróneos porque no comprenden ni aprovechan las fuerzas conjuntas de propósito moral de las acciones de cambio (Fullan, p. 57).

Sólo en un escaso número de casos los centros educativos y sus respectivas comunidades establecen vínculos sólidos, a partir de algún proceso de innovación liderado por el profesorado (generalmente, una parte del claustro) o un grupo de familias. En este artículo comentaremos el caso de dos centros innovadores en lo relativo a la participación de la comunidad, que hemos explorado mediante estudios de caso, siguiendo técnicas propias de las metodologías cualitativas, observación y entrevistas, principalmente.

Hay diversas vías de participación de las familias, pero es en este artículo vamos a centrar la atención en (1) la Acción Tutorial y (2) la Apertura de Centros a la Comunidad. La Acción Tutorial presta atención a la persona global y nos recuerda que en educación no hay funciones exclusivas: todas son compartidas (San Fabián, 2006, p. 141). Prestamos atención a la Apertura de Centros a la Comunidad porque a través de ésta se puede hacer efectiva la participación de todas las personas implicadas en la educación en el centro escolar.

A continuación pasamos a comunicar los resultados que la investigación educativa nos ha aportado, a partir de los resultados de dos estudios de casos, en los que la participación de la comunidad educativa y la reflexión sobre la práctica docente son los principales protagonistas en los procesos de innovación y cambio y donde la acción tutorial y la apertura 
de centros a la comunidad son los medios que nos brinda la organización educativa para llevar a cabo estos procesos. Para ello, vamos a presentar los testimonios de profesorado, alumnado y familias recogidos en las entrevistas realizadas en el estudio.

\section{Estudio de casos}

En concreto, hemos estudiado dos centros públicos de Educación Infantil y Primaria diferentes en sus contextos, uno es asturiano y el otro cántabro, y en sus acciones: el primero desarrolla la relación con la comunidad a través del Proyecto de Apertura del Centro a la Comunidad y de la Acción Tutorial, el segundo desarrolla la relación con la comunidad a través de una comisión de familiares y una red de voluntariado que acude a la escuela a realizar "grupos interactivos". Además en el primer caso el cambio se produce por parte de un docente (un profesor-tutor del tercer ciclo) que encabeza y lidera el proceso, mientras en el segundo el cambio se inicia con una docente (una profesora-tutora del tercer ciclo) envolviendo en el proceso a otros cinco profesores más de la escuela.

En ambos casos se podría decir que se implementan conscientemente acciones contando con familias, alumnado, profesorado y otras personas del entorno próximo: asociaciones culturales, bibliotecas públicas, personal de administración y servicios del centro, ciudadanos del barrio, etc. para favorecer la relación entre el centro y su comunidad. También en ambos casos se cuenta con el apoyo del equipo directivo.

Para proceder al estudio de ambos centros se han desarrollado dos estudios de caso siguiendo una metodología etnográfica, realizándose observación participante y entrevistas con informantes clave a lo largo de los pasados cursos. A continuación se comentan los principales resultados, mostrando cómo en todos los casos se están buscando espacios para la relación escuela-comunidad.

En el caso del centro educativo asturiano, se trata de un centro situado a las afueras de Oviedo. Se trata de un centro de doble línea con un profesorado cuya media de edad supera los cincuenta años, que ha visto incrementar su porcentaje de alumnado inmigrante en los últimos años hasta un $25 \%$.

Para estudiar la relación de éste centro con la comunidad se han analizado dos vías fundamentales y destacadas: la tutoría que desarrollaba uno de sus maestros, el cual estaba profundamente concienciado con la tarea y el desarrollo del Plan de Apertura del Centro a la Comunidad, que coordinaba este mismo profesor, dándole especial protagonismo a las familias y la comunidad educativa del centro en general.

Por lo que respecta a la tutoría cabe destacar la implicación del profesor en su positiva relación con la comunidad educativa:

1. Siendo una persona cercana y amistosa que aprovechaba cualquier oportunidad para entablar conversación con las familias de su alumnado y con la comunidad en general.

Antigua alumna: "Siempre fue muy agradable con todas las familias de sus alumnos". Entrevistadora. "¿Y en qué lo notabas tú?"

Antigua alumna. "Lo notaba en su forma de hablar, de tratar a la gente".

Cualquier docente que se proponga relacionarse con la comunidad educativa de su centro debe superar los prejuicios relativos al contexto socio-económico del alumnado y familias y abrir cauces para la comunicación. Como afirma esta antigua alumna en 
una entrevista, el trato afable y la forma de referirse a las familias de este profesional era diferente de las habituales y su repercusión es muy positiva.

2. Realizando reuniones frecuentes con las familias más predispuestas de su alumnado y provocándolas con aquellas más difíciles.

Antiguo alumno. "Gracias a él se preocupó mi padre de mí por primera vez y fue a la escuela. Nunca antes había ido a la escuela a nada, ni a una reunión ni a nada y un día mi tutor lo llamó y fue, por primera y única vez".

Las reuniones individuales y en grupo siempre son un recurso que los docentes pueden emplear. Como plantea este alumno, gracias a una llamada del docente y una reunión personal, se puede implicar a un familiar en la formación de un hijo, incluso en dinámicas más amplias a nivel de centro.

3. Estableciendo un horario de atención a familias en el tiempo posterior a las clases y ampliando éste cuando la situación lo requiere. A continuación se muestra el testimonio de una madre cuyo hijo fue alumno durante varios cursos del citado profesor-tutor:

Madre. "El jueves a la hora de visita yo estoy trabajando y no puedo ir. Él me recibe cuando pueda. Sé que hay alumnos que no va nadie en todo el año. Él intenta estar en contacto con las familias, y a las reuniones intenta que vengamos los máximos posibles, le da rabia que seamos pocos. Y siempre le digo que ante cualquier problema no dude en llamarme".

Un gesto como establecer un horario de atención a familias conocido por todos (alumnado, familias y profesorado) y su apertura, si fuese necesario, para atender a quienes tengan más limitaciones horarias puede contribuir a cohesionar centro y comunidad.

4. Defendiendo la relevancia de que las familias estén próximas al centro en los diferentes foros, en reuniones formales como los claustros o los ciclos, así como en todo tipo de encuentros informales, incluso cuando una parte del profesorado del centro se resistía a ello.

Así valora una profesora especialista el empeño de este docente en relacionarse con las familias del centro educativo.

Profesora especialista 1. “Si veo que favorezca las vías de comunicación con las familias? Desde luego que sí. Más que ningún otro profesor que yo haya conocido hasta ahora. En muchísimos aspectos: no sólo en tener contactos con ellos para hablar cuestiones puntuales, sino que todas las actividades que yo veo que organiza, siempre deja buena cuenta para que participen las familias y una de sus preocupaciones, en las actividades que compartimos siempre fue el rechazo del resto del profesorado a abrir esa puerta de participación a las familias".

Entrevistadora. "¿Te ha dicho que el resto del profesorado no está dispuesto a abrir la puerta de participación a las familias?"

Profesora especialista 1. "Cierta parte del profesorado sí, la mayoría no".

Profesor especialista 2. "Se ve. No hace falta que nadie lo diga". 
El profesorado más innovador en materia de apertura del centro a las familias tenía que permanentemente luchar contra la tendencia negativa mostrada por una parte importante del profesorado del centro.

5. Proponiendo actividades que permitan abrir vías de comunicación con las familias y otros miembros de la comunidad escolar.

Madre antigua alumna. "Una de las cosas en las que se molestó mucho de aquella fue en abrir el centro a la comunidad, a los padres, preparaba charlas y conferencias. Creo que antes no se hacía. La primera que vi, fue él el que lo empezó a mover, y es complicado mover a los padres porque si no tienen que trabajar tienen que hacer otra cosa, pero él siempre trataba de buscar algún tema que te enganchara o alguna cosa que te interesara referente a ellos. Eran muy interesantes. Siempre sacas cosas, conclusiones y aprendes a saber por qué camino tienes que ir con ellos, porque es difícil educarlos. No puedes ser ni muy buena ni muy mala. Ni puedes ser su amigo, tienes que ser su madre".

Como se puede deducir de las cinco ideas anteriores, la acción tutorial abre vías de relación familia-centro muy importantes, que la comunidad valora positivamente como hemos podido leer en los testimonios de las personas implicadas.

La segunda vía que ha arbitrado este docente en su centro para favorecer la relación del centro educativo con su comunidad ha sido la elaboración de un Plan de Apertura del Centro a la Comunidad (PAAC). Pese a que el objetivo de los PAAC en las escuelas es "abrir el centro a toda la comunidad", el foco principal sigue siendo el alumnado, como lo ha sido tradicionalmente, ofreciéndosele actividades extraescolares, talleres, clases de refuerzo, etc. Solamente en contados centros se organizan, al amparo de este plan, actuaciones dirigidas a la comunidad escolar entendida en sentido amplio. Tal es el caso de este centro, que también desarrollaba una Escuela de Padres, y un Club de Lectura para adultos, abierto a la participación de toda la comunidad, en el que participaban familiares, profesorado de ese y otros centros, profesionales de la red de bibliotecas públicas, alumnado de prácticas de Magisterio, una profesora universitaria, etc.

En una entrevista con el director del centro éste comentó:

Entrevistadora. "¿Así que el proyecto de apertura de centros se dirige principalmente a actividades para los alumnos? Es apertura, pero es para alumnos. ¿A las familias no se las contempla directamente?"

Director. "Menos, menos, menos. Hay una menor proporción, si".

Entrevistadora. "¿Es cosa del coordinador del PACC que se enfatice también en las familias?"

Director. "Claro. Sí, sí, sí. Yo no conozco otros proyectos de apertura, pero me da la impresión que está todo dirigido a alumnos en los otros centros. Y él con la familia lo intentó con las conferencias, lo intentó con la fiesta de fin de curso, que bueno, es una chorrada, pero ahí está. Hay una manera de conseguir la implicación con las familias y es esa. Ahí está. Y ahí dio como un clavo. Hasta el final peleando". 
Podríamos decir que, en estas dos medidas, la acción tutorial y los PACC, los centros educativos que deseen implicarse tienen dos aliados importantes para acercar escuela y comunidad.

En el caso del centro cántabro, situado en el casco antiguo de Castro Urdiales, se trata de un centro de doble línea con un profesorado cuya media de edad ronda los cuarenta años que, como el otro, ha comenzado a matricular algunos alumnos inmigrantes en los últimos años, si bien el porcentaje de los mismos en el centro es mínimo.

Para estudiar la relación del centro con la comunidad se han analizado dos vías fundamentales y destacadas: la experiencia de crear "grupos interactivos" en seis aulas y la de crear una comisión de familias que se reúna periódicamente para mejorar la comunicación del centro con las mismas.

Este centro ha venido dando pasos en los anteriores cursos con el objetivo de configurarse como comunidad de aprendizaje (Elboj, Puigdellívol, Soler y Valls, 2006). Dado que inicialmente no se contaba con la aprobación de un porcentaje significativo del claustro para iniciarse en este proceso se optó por emprender prácticas propias de estos centros: grupos interactivos y tertulias literarias (Álvarez Álvarez y Larrinaga Iturriaga, 2012).

Los grupos interactivos son una forma diferente de enseñanza en el aula y consisten en hacer grupos dentro de la clase de alumnado con diferente nivel de conocimientos, género, etnia, etc. para acelerar el aprendizaje de todos. En estos grupos entran a colaborar voluntarios/as de la comunidad educativa (madres y padres, profesores jubilados, abuelos y abuelas, estudiantes de magisterio en prácticas, etc.) y se hacen tareas que suelen ser de repaso, de tal manera que en el grupo es probable que algunos alumnos ya sepan resolverlas mientras otros aún muestran dificultades. En el tiempo de una o dos clases se organizan tantas actividades para realizar como grupos hay en el aula y los alumnos van rotando por los grupos resolviendo las diferentes actividades con el apoyo de las personas voluntarias, que no se encargan de "enseñar" cómo se resuelven los ejercicios, sino de contribuir a que se desarrolle una trama de interacciones dialógicas entre los miembros del grupo para que todos/as los/as niños/as del grupo aprendan a resolver las tareas propuestas, colaborando entre sí (Flecha, 1997).

Las principales oportunidades que hemos detectado en los grupos interactivos son:

1. El alumnado se enriquece con las aportaciones de las personas voluntarias que entran a colaborar en ella. Así lo ve una alumna y un alumno del centro:

Alumna. "Me gusta trabajar en grupos interactivos porque hablamos de cómo se resuelve el ejercicio hasta que lo hacemos y nos ayudamos entre nosotros y el voluntario también nos va guiando y nos ayuda a aprender".

En línea con este cambio entroncan otros que se han ido generando con posterioridad y demuestran que es posible trabajar de manera solidaria entre maestros/as y familias. Este curso la dirección del centro ha realizado un notable esfuerzo por acercarse a las familias de todos los grupos creando una comisión de familiares que mantenga reuniones periódicas (a ser posible todos los meses), para intervenir sobre todas aquellas carencias o problemas que se vayan detectando. Para ello se han realizado reuniones al comienzo del curso con las familias de todos los grupos y se ha elegido un delegado de cada uno, configurándose así una comisión de familias comprometida con la mejora escolar de la escuela. 
2. Se genera un vínculo de comunicación fluido y cercano entre voluntariado y profesorado y ambos aprenden entre sí.

Voluntario. "Ahora valoro más el trabajo de los maestros porque veo lo duro que es. Yo estoy una hora y media a la semana y pienso en la maestra que está todos los días a todas horas y me compadezco de ella".

El voluntariado respeta cada vez más la dureza del trabajo de los docentes y éstos agradecen su colaboración desinteresada de los voluntarios en el centro, sin los cuales no es posible hacer los grupos interactivos.

3. El voluntariado agradece poder participar en la educación de los niños y éstos se lo agradecen a las personas voluntarias, generando lazos afectivos.

Voluntaria. "Me gusta ver la evolución de los niños cada semana y saber que están aprendiendo mientras se lo pasan muy bien. Sería bueno que se implicaran cuantas más personas porque los niños aprenden mucho más asi".

Alumno. "Me gustaría conocer a todos los voluntarios de la escuela porque los que conozco me parecen muy majos y son muy buenos porque nos ayudan siempre”.

Para alumnado y profesorado los vínculos generados son reconfortantes.

1. El voluntario y el profesorado se apoyan en el aula para evitar la exclusión del alumnado con más dificultades, ofreciéndole al alumnado el apoyo que requiera.

Voluntaria. "Nadie queda fuera. Todos hacen la actividad. Se ayudan entre sí y los ayudo yo también y todos aprenden. Así se atiende a todos y todas. No se deja fuera a nadie".

Haciendo balance del trabajo de apertura de estas dos escuelas, atendiendo al surgimiento de la innovación y a las personas implicadas, las dos dimensiones más importantes en estos procesos de cambio (Rivas Borrell, 2007), podemos sintetizar las propuestas de la siguiente manera: 
Tabla 1: Síntesis de la apertura a la comunidad que realizan los dos casos examinados

\begin{tabular}{|l|l|l|l|l|}
\hline & Surgimiento & Vía de relación & Personas implicadas & Contexto \\
\hline & $\begin{array}{l}\text { Un docente en colabora- } \\
\text { ción con el equipo direc- } \\
\text { tivo }\end{array}$ & $\begin{array}{l}\text { Acción tutorial } \\
\text { zado, familias de su } \\
\text { grupo y familias del } \\
\text { centro. }\end{array}$ & $\begin{array}{l}\text { Centro (más } \\
\text { allá del aula) }\end{array}$ \\
\cline { 2 - 5 } & $\begin{array}{l}\text { Un docente en colabora- } \\
\text { ción con el equipo direc- } \\
\text { tivo y el AMPA }\end{array}$ & $\begin{array}{l}\text { Proyecto de Aper- } \\
\text { tura del Centro a } \\
\text { la Comunidad }\end{array}$ & $\begin{array}{l}\text { Profesor, alumnado, } \\
\text { equipo directivo, fami- } \\
\text { lias, AMPA y personas y } \\
\text { entidades del barrio. }\end{array}$ & $\begin{array}{l}\text { Centro en su } \\
\text { totalidad }\end{array}$ \\
\hline $\begin{array}{l}\text { Una docente, en cola- } \\
\text { boración con el equipo } \\
\text { directivo }\end{array}$ & $\begin{array}{l}\text { Grupos interac- } \\
\text { tivos }\end{array}$ & $\begin{array}{l}\text { Tutores, alumnado, fa- } \\
\text { milias, personas volunta- } \\
\text { rias (de la comunidad lo- } \\
\text { cal). }\end{array}$ & $\begin{array}{l}\text { Centro (seis } \\
\text { aulas implica- } \\
\text { das) }\end{array}$ \\
\cline { 2 - 5 } & $\begin{array}{l}\text { El equipo directivo, en } \\
\text { colaboración con los } \\
\text { tutores y familias }\end{array}$ & $\begin{array}{l}\text { Comisión de fa- } \\
\text { milias }\end{array}$ & $\begin{array}{l}\text { Equipo directivo y fami- } \\
\text { lias de todos los cursos. }\end{array}$ & $\begin{array}{l}\text { Centro en su } \\
\text { totalidad }\end{array}$ \\
\hline
\end{tabular}

Ambas experiencias nos demuestran que la participación de las familias en la Acción Tutorial y la Apertura de Centros a la Comunidad son acciones que pueden contribuir a los procesos de innovación y cambio en las organizaciones, y esta reflexión sobre la práctica al mismo tiempo contribuye al aprendizaje de la propia organización educativa. En ambos casos, los centros educativos han apostado por relacionarse con la comunidad educativa y lo han conseguido, siguiendo fórmulas diferentes, que perfectamente podrían ser complementarias, contribuyendo a favorecer un mayor diálogo entre el centro escolar y su entorno, humanizándose a sí mismas cada día un poco más. Cada una de las estrategias seguidas por las escuelas es interesante y valiosa. Aunque, en ocasiones, en el plano teórico demos por superadas muchas tendencias, la realidad nos muestra día tras día que en los centros educativos aún quedan muchas vías de relación con la comunidad sin explorar y/o explotar, tratando de integrar todo tipo de personas.

\section{Reflexiones finales}

Si queremos innovar, no es necesario partir de la nada, puesto que las organizaciones educativas disponen de medios y recursos para canalizar la participación de las familias y de la comunidad educativa en general en los centros. Hay que seguir investigando en la dirección de cómo desde la organización escolar se pueden articular y mejorar estos procesos.

La participación de las familias y de la comunidad educativa en general es en sí un proceso de innovación y cambio en las organizaciones educativas y puede contribuir a la mejora y cambio de la escuela. La acción tutorial es un ámbito perfecto para fomentar la participación de las familias y hacerles partícipes de la educación de sus hijos/as, así como la apertura de los centros a la comunidad. 
Es necesario reflexionar sobre la práctica, mantener los ojos abiertos, escuchar al alumnado, profesorado y familias y a toda la comunidad en general. Las innovaciones y cambios en las organizaciones educativas, han de realizarse en colaboración, con profundidad, con apoyaturas teóricas, y han de estar pensadas para que sean duraderas en el tiempo y proyectados en diferentes ámbitos con la participación de toda la comunidad educativa.

$\mathrm{Y}$, por supuesto, es fundamental continuar investigando, y que la propia investigación aporte información relevante que nos permita conocer mejor la realidad y formular propuestas de mejora. Es fundamental que todos los docentes reflexionen sobre la práctica docente y favorezcan procesos de cambio y mejora en las organizaciones.

Como apuntan Hargreaves y Fink (2006) los cambios que necesitamos para el aprendizaje profundo y duradero de los estudiantes requieren procesos sostenibles, democráticos y justos. Han de inspirarse en ciertos principios como amplitud, profundidad, continuidad, diversidad, justicia, disponibilidad de recursos y conservación de la memoria. Merecen ser proyectados sobre diversas esferas de influencia recíproca entre la escuela y la comunidad, el sistema educativo en su conjunto y los movimientos sociales.

Como ya hemos comentado con anterioridad, la escuela necesita cambios, pero estos no han de ser puntuales, sino que han de hacerse en profundidad y han de ser duraderos y tener permeancia en el tiempo. Como apuntan Hargreaves, Earl y Ryan (1998, p. 253) si queremos mejores perspectivas para los adolescentes actuales y un futuro mejor para el mundo que heredará mañana, no cabe la menor duda de que nuestras escuelas necesitan efectuar cambios fundamentales y de largo alcance.

La participación es un punto de partida y como nos recuerda San Fabián (2004), seguramente la escuela no es una réplica del sistema político en miniatura, ni un ejemplo reducido a escala de la sociedad en la que vivimos, pero sí inicia en ella. En la escuela se dan unas relaciones humanas complejas que ofrecen oportunidades suficientes para poner en práctica los principios democráticos. Posiblemente la participación no sea el valor supremo de un sistema social, pero sí es su mejor instrumento para acceder a otros valores fundamentales como son la justicia, la creatividad, la igualdad, la tolerancia o la solidaridad; es decir, es su caldo de cultivo. 


\section{Referencias}

Álvarez Álvarez, C. \& Larrinaga Iturriaga, A. (2012). Iniciando grupos interactivos en la comunidad cántabra. Aula de Innovación Pedagógica, 210, 53-57.

Bolívar Botía, A. (2001). Los centros educativos como organizaciones que aprenden: una mirada crítica. Revista Digital de Nuevas tecnologías, 18. Consultado en: http://contexto-educativo.com.ar/2001/4/nota-04.htm Fecha de consulta: 16-04-2012.

-. (2000). Los centros educativos como organizaciones que aprenden. Promesas y realidades. Madrid: La Muralla.

Bolívar Ruano, M.R. (2012). La cultura de aprendizaje de las organizaciones educativas. Instrumentos de diagnóstico y evaluación. Revista Iberoamericana sobre calidad, eficacia y cambio en la educación. Vol. 10, 1, Consultado en: http://www.rinace.net/reice/numeros/arts/vol10num1/art9 htm.htm Fecha de consulta: 12- 09-2012.

Elboj, C., Puigdellívol, I., Soler, M. \& Valls, R. (2006). Comunidades de aprendizaje. Transformar la educación. Barcelona: Graó.

González González, M. T. (Coord.). (2011). La atención, el cuidado, las relaciones y la responsabilidad del centro escolar con los estudiantes. En González M.T.; Escudero J.M.; Nieto J. M \& Portela, A. Innovaciones en el gobierno y la gestión de los centros escolares. (41-58). Madrid: Editorial Síntesis S.A.

Fernández Enguita, M. (2006). La Corporación contra la comunidad. Revista Cuatrimestral del Consejo Escolar del Estado, 3, 18-22.

Flecha, R., Padrós, M. \& Puigdellívol, I. (2003). Comunidades de Aprendizaje: transformar la Organización Escolar al servicio de la Comunidad. Organización y gestión educativa, 5, 4-8.

Flecha, R. (1997). Compartiendo palabras. El aprendizaje de las personas adultas a través del diálogo. Barcelona: Paidós.

Fullan, M. (2002). Las fuerzas del cambio. Explorando las profundidades de la reforma educativa. Madrid: Alkal.

Hargreaves, A. y Fink, D. (2006). Estrategias de cambio y mejora en educación caracterizadas por su relevancia, difusión y continuidad en el tiempo. Revista de Educación, 339, 43-58.

Hargreaves, A., Earl, L. \& Ryan, J. (1998). Una educación para el cambio. Reinventar la educación para los adolescentes. Barcelona: Octaedro.

Imbernón, F. \& Martínez Bonafé, J. (2008). Innovar en la teoría y en la práctica. Cuadernos de pedagogía, 385, 62-65.

López Yáñez, J. (2003). Abriendo la caja negra. Una perspectiva sistémica sobre el cambio en las organizaciones educativas. XXI Revista de Educación, 5, 139-155.

Martín Bris, M. (2000). Clima de trabajo y organizaciones que aprenden. Educar, 27, 103-117.

Martínez González, R.A. \& San Fabián, J. L. (2002). Autoevaluación de la cultura participativa del centro. Organización y Gestión Educativa, 4, 19-26. Consultado en: http://www.unioviedo.es/COSPRAS/documentos/mod1/autoevaluacionParticipacion.pdfFecha de consulta: 10-04-2012.

Rivas Borrell, S. (2007). La participación de las familias en la escuela. Revista Española de Pedagogía, 238, 559-574.

San Fabián J.L. (2011). El papel de la organización escolar en el cambio educativo: la inercia de lo establecido. Revista de Educación, 356, 41-60. Consultado en: http://www.revistaeducacion.educacion.es/re356/re356_02.pdf Fecha de consulta: 25-04-2012.

-. (2006). Participación de las Familias y Acción tutorial: Dos puertas para el cambio. En García Albadalejo, A. (Coord.). Participación de las Familias en la Vida escolar: Acciones y Estrategias. (131-148). Madrid: Ministerio de Educación y Ciencia. Secretaría General de Educación. 
—. (2005). Participar en las organizaciones Educativas: un ejercicio de ciudadanía. En VV.AA. Escuelas para la democracia. Cultura, organización y dirección de instituciones educativas (177200). Cantabria: Consejería de Educación del Gobierno de Cantabria.

—. (1996). ¿Pueden aprender las escuelas? La autoevaluación al servicio del aprendizaje organizacional. Investigación en la escuela, 30, 41-51.

Santos Guerra, M. A. (2005). La escuela que aprende. Retos, dificultades y esperanzas. En VV.AA. Escuelas para la democracia. Cultura, organización y dirección de instituciones educativas (157-176). Cantabria: Consejería de Educación del Gobierno de Cantabria.

-. (2000). La escuela que aprende. Madrid: Morata.

Tsang, E.W.K. (1997). Organizational learning and the learning organization: A dichotomy between descriptive and prescriptive research. Human Relations, 50 (1), 73-89.

\section{Sobre las Autoras}

Carmen Álvarez Álvarez: es profesora en el área de Didáctica y Organización Escolar en la Universidad de Cantabria y coordinadora de la asignatura Organización del Centro Escolar. Es licenciada y doctora en Pedagogía por la Universidad de Oviedo donde previamente también impartió docencia en el Departamento de Ciencias de la Educación. Su trayectoria investigadora se enmarca en la relación teoría-práctica en la educación, la formación del profesorado, la educación en valores, las metodologías dialógicas de enseñanza y la animación a la lectura. Ha publicado artículos sobre educación en prestigiosas revistas, ha participado en diversos congresos de carácter nacional e internacional y ha colaborado en diversas investigaciones nacionales sobre educación del Plan Nacional I+D+I.

Maria Verdeja Muñiz: es licenciada en Pedagogía, por la Universidad de Oviedo. Ha realizado el Programa de Doctorado; Pedagogía: Evaluación y procesos educativos, y ha obtenido el Diploma de Estudios Avanzados (D.E.A), con el trabajo de Investigación titulado: Clima de Convivencia y Acción tutorial: Un estudio de caso en la Educación Secundaria Obligatoria. Actualmente trabaja como profesora asociada en la Universidad de Oviedo, Departamento de Ciencias de la Educación en el área de Didáctica y Organización Escolar, y está escribiendo la Tesis Doctoral. 